\title{
Variable-Order Iterative LMS Adaptive Filtering Algorithm
}

\author{
Nie Xiangju ${ }^{1}$ Miao Xinglei ${ }^{2}$, Qiao Shuang ${ }^{1}$, and Ge Hongjun ${ }^{1}$ \\ ${ }^{1}$ Heilongjiang Institute of Technology, Harbin, China \\ ${ }^{2}$ Harbin First Machines Manufacturing Limited Company, Harbin, China
}

\begin{abstract}
This paper introduces the Fractional tap-length least mean square (FTLMS) algorithm with fractional order, Analysis the effect of the performance for the FTLMS algorithm convergence by the iterative parameters in this algorithm.A new variable parameter FTLMS (VP-FTLMS) is proposed to reduce the output error of the system with the order of the filter. The algorithm proposed in this paper is simulated and validated in both high noise and low noise environment, and compared with the FTLMS algorithm with different filter order iterative parameters. The simulation results show that the proposed VP-FTLMS algorithm has faster convergence rate and smaller steady-state oscillation than the FTLMS algorithm, and it can also obtains a small excess mean square error (EMSE).
\end{abstract}

Keywords: adaptive filter, variable tap-length, variable adaptive parameter

\section{Introduction}

The order of the filters is usually unknown at the time of the filter design. If the order of the filter we chose is too small, it will bring large error to the design of the filter. However, if the order we chose is too large, it will not only bring a large amount of computation, but also lead to algorithm error. This paper introduces the idea and characteristics of three classical variable-order algorithms [1]. In this paper, the steady-state analysis of the fractional-order variation-order algorithm is used to derive the theoretical expression of the steady-state order, and the basis of the algorithm selection is given.

In order to solve the problem that the order of the filters is unknown in practical applications, we propose a variable order algorithm such as Segmented Filter LMS (SF-LMS), Gradient Descent LMS, (GD-LMS ) and Fractional Tap-length LMS (FTLMS). SF-LMS algorithm and GD-LMS algorithm both assume the filter order is an integer, FTLMS algorithm eliminates the order of integer constraints, increases the flexibility of the algorithm and its computational complexity is small, so it can quickly adapt to the changes in the environment [2].

\section{Fractional Order Number Change Order Number LMS Algorithm}

In most LMS algorithms, they all assuming that the order of the filter is an integer, which limit the flexibility of the filter design and also bring some errors. To solve this problem, the proposed algorithm eliminates the restrictions that the order should be integer, and the introduction of the concept of fractional order. We call this algorithm Fractional Tap-length LMS (FTLMS) algorithm, referred to as the FTLMS algorithm [3]. In this paper, we use the system identification model in the under picture as an example to introduce FTLMS.In this system model, the desired signal $d(n)$ can be expressed as

$$
d(n)=X_{\text {Lopt }}^{T}(n) W_{\text {Lopt }}+t(n)
$$

where $W_{\text {Lopt }}$ is the unknown system weight coefficient, $L_{o p t}$ is the optimal filter order that need to determine, $X_{\text {Lopt }}(n)$ is the input signal matrix."

\footnotetext{
* Nie Xiangju(1980-,Female,Master,Lecturer, Graduated from Heilongjiang University of science and technology, Major in electrical engineering and automation,Email:55049822@qq.com)
} 


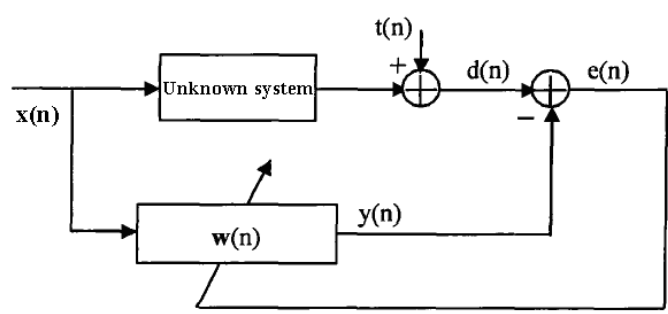

Fig. 1: Model of system identification.

Assuming that the steady-state filter order converges to a fixed value $\mathrm{L}$, then the segment error is defined as the error of the front $\mathrm{M}$ elements of the filter of order $\mathrm{L}$, which is

$$
e_{M}^{(L)}(n)=d(n)-W_{L, 1: M}^{T} X_{L, 1: M}(n)
$$

where $1 \leq M \leq L, \quad W_{L}$ and $X_{L}(n)$ denote the steady-state filter coefficients and the input vectors respectively, $W_{L, 1: M}$ and $X_{L, 1: M}(n)$ respectively denote the front M elements of $W_{L}$ and $X_{L}(n)$.In the FTLMS algorithm, we defined the cost function of the filter order as the square of the segment error, which is

$$
\xi_{M}^{(L)}=E\left\{\left(e_{M}^{(L)}(n)\right)^{2}\right\}
$$

The FTLMS algorithm needs to find the smallest $L$ to make it satisfy $\xi_{L-\Delta}^{(L)}-\xi_{L}^{(L)} \leq \varepsilon$, where $\Delta$ is a positive integer less than $\mathrm{L}, \quad \varepsilon$ is a small positive integer that is required by the system. Then the smallest $\mathrm{L}$ that satisfied the above expression is selected as the optimum filter order [4].

In the FTLMS algorithm, the filter order is defined as a fractional order $l_{f}(n)$, The iteration process of this fraction is:

$$
l_{f}(n+1)=\left(l_{f}(n)-\alpha\right)-\gamma\left[\left(e_{L(n)}^{(L(n))}(n)^{2}-\left(e_{L(n)-\Delta}^{(L(n))}(n)\right)^{2}\right]\right.
$$

where: $\gamma$ Is the iterative step of the order, In order to differentiate the iterative steps of the weight coefficients of the filter, we called $\gamma$ as the iterative parameter of order in this paper. $\alpha$ is a positive leakage parameter whose function is to avoid the problem that the iterative order tends to a value much larger than the optimal order and can not converge to the optimal order, and $\alpha<<\gamma$, This iterative method is similar with the way that LMS algorithm weight coefficient iterative, so called LMS way of order iteration [5].

When the cumulative number of changes in the fractional order $l_{f}(n)$ exceeds a certain threshold, the filter order is rounded as shown in the following equation:

$$
L(n+1)=\left\{\begin{array}{c}
\left\lfloor l_{f}(n)\right\rfloor\left|L(n)-l_{f}(n)\right|>\delta \\
L(n) \text { otherwise }
\end{array}\right.
$$

- |represents to select a integer that nearest to $l_{f}(n), \delta$ Is a small integer threshold. If $\delta=1$, The filter order is converged to the optimal solution that satisfied (3) $L_{o}=L_{o p t}+\Delta$, If $\delta \neq 1$, The filter order is converged to $\left(L_{o}-\delta, L_{o}+\delta\right)$.

At the same time as the filter order is updated, the filter weight coefficients are updated as follows:

$$
W_{L(n)}(n+1)=W_{L(n)}(n)+\mu e_{L(n)}^{(L(n))}(n) X_{L(n)}(n)
$$

where $W_{L(n)}$ and $X_{L(n)}$ represent the weight coefficients of the filter and the input vector respectively, $\mu$ is the filter coefficient iterative step size [6].

\section{Variable-Order LMS Algorithm for Iterative Parameters}

In view of the FTLMS algorithm is an LMS way of filter order update algorithm, the order iterative process and LMS algorithm has similar characteristics. From the analysis above, $\gamma$ is the step size of the filter iterations. To differentiate the iterative steps of the LMS algorithm, we will call $\gamma$ iterating parameters of the filter order iterations. Obviously, the bigger the filter order $\gamma$, the faster the convergence will be, however, this will lead to a larger order of steady-state oscillation; the smaller $\gamma$ is the smaller the steadystate shock, but the filter order will slow down the convergence rate. And once the order of the adaptive filter 
to less than the unknown order of the filter, will produce a large additional error [7]. Therefore, the value of $\gamma$ is a contradiction amount between the convergence rate of the filter order and steady-state shock.

In this paper, a variable order LMS algorithm with variable iteration parameters is proposed, and the smaller steady-state oscillations are obtained with smaller values after the order reaches steady-state [8]. In addition, the steady-state use the small iterative parameters to reduce the steady-state shock, it is can possible avoid the additional error caused by the order decreasing to the optimal order. In the following analysis, we will propose variable iterative parameters of the variable order algorithm, which we referred to VP-FTLMS algorithm (Variable parameter fractional tap-length LMS algorithm).

\section{Analysis of VP-FTLMS Algorithm}

From the above analysis we can see, we need to find an appropriate amount to change the iterative parameters $\gamma$, so the variable-order algorithm has faster convergence speed and smaller steady-state error[9]. From the iterative process of the LMS algorithm, the instantaneous error can be expressed as $e(n)=\zeta(n)+t(n)$, it contains two components: system noise $t(n)$ and iterative noise $\zeta(n)$.Assumption that $\zeta(n)$ and $t(n)$ are irrelevant, then the following relationship holds

$$
E\left\{e^{2}(n)\right\}=E\left\{\zeta^{2}(n)\right\}+E\left\{t^{2}(n)\right\}
$$

where $E\left\{e^{2}(n)\right\}$ for the mean square error MSE, $E\left\{\zeta^{2}(n)\right\}$ for extra mean square error EMSE, $E\left\{t^{2}(n)\right\}$ for System Noise Mean Square. In view of EMSE has the characteristics that large in the initial stage of the system and approaching to zero when the system in the steady state. Which is a good reflection of the adaptive filter iterative process, we use the EMSE to control the iterative parameters of the filter order. In practice the EMSE is estimated to be:

$$
E\left\{\zeta^{2}(n)\right\} \approx \hat{e}^{2}(n)-\sigma_{t}^{2}
$$

where $\hat{e}^{2}(n)$ is the variance estimate of the output error, $\sigma_{t}^{2}$ is the system noise variance.

By using the estimated characteristic of EMSE, we proposed VP-FTLMS algorithm in this paper and it is described as follow:

$$
\begin{gathered}
\hat{e}^{2}(n)=\rho \hat{e}^{2}(n-1)+(1-\rho) * e^{2}(n) \\
g=\frac{\left|\hat{e}^{2}(n)-\sigma_{t}^{2}\right|}{\left|\hat{e}^{2}(n)-\sigma_{t}^{2}\right|+\sigma_{d}^{2}-\sigma_{t}^{2}} \\
\gamma(n)=\gamma_{\max } * g \\
l_{f}(n+1)=\left(l_{f}(n)-\alpha\right)-\gamma(n)\left[\left(e_{L(n)}^{(L(n))}(n)\right)^{2}-\left(e_{L(n)-\Delta}^{(L(n))}(n)\right)^{2}\right]
\end{gathered}
$$

where $\mathrm{g}$ is a change factor defined according to EMSE, the molecule is the Instantaneous EMSE, the denominator is the sum of the initial EMSE and the instantaneous EMSE. In order to overcome the transient error of the shock, we make the instantaneous error of the system smoothed in the algorithm. As when near the steady state, $\hat{e}^{2}(n)$ will be very close to $\sigma_{t}^{2}$, in order to prevent the estimated value is not accurate enough that make $\hat{e}^{2}(n)-\sigma_{t}^{2}<0$, absolute operation is used. The $\rho$ in formula (9) is a smoothing parameter of less than 1 and close to 1 , used to estimate the variance of the output error; The $\gamma_{\max }$ in formula (11) is a certain multiple of $\gamma$.

In the original FTLMS algorithm, the iterative step of the coefficient $\mu$ is a constant. In order to speed up the iterative speed of the system coefficients, in the VP-FTLMS algorithm that proposed in this paper, the coefficient iteration step $\mu$ adopts variable step size too. The iterative parameters of the coefficient iterative step are different from those of the order. The iterative process is simultaneous but independent. The method of updating the coefficient iterative step size is

$$
\mu(n)=\frac{\mu^{\prime}}{(L(n)+2) \sigma_{x}^{2}}
$$


where $\mu^{\prime}$ is a constant, $\sigma_{x}^{2}$ is the variance of the input signal. Then the new system coefficient update equation is

$$
W_{L(n)}(n+1)=W_{L(n)}(n)+\mu(n) e_{L(n)}^{(L(n))}(n) X_{L(n)}(n)
$$

where $L(n)$ is obtained by the VP-FTLMS algorithm proposed in this paper, the fractional order of the change in the accumulation of more than the threshold $\delta$ obtained after the rounded.

\section{Comparison and Simulation of VP-FTLMS and FTLMS Algorithm}

The performance evaluation of the LMS algorithm depends on the convergence rate of the order, the steady-state oscillation and the convergence rate and steady-state value of the EMSE. In this paper, the VPFTLMS algorithm that proposed will be simulated in both high-noise and low-noise environments [10]. And compare the convergence performance with FTLMS algorithm under different order iterative parameters to reflect the superiority of the algorithm proposed in this paper. The simulation results of VP-FTLMS algorithm in low noise and high noise environment are given and compared with FTLMS algorithm.

\subsection{Low Noise, $S N R=20 d B$}

The simulation settings in the low-noise environment are as follows: The parameter $\delta$ is set to $2, \mu$ ' set to 0.5 , Leakage factor $\alpha$ set to $0.005, \Delta$ set to 20.In the VP-FTLMS algorithm proposed in this paper, smoothing parameters $\rho=0.99$.In order to compare the impact of different values $\gamma$ for the FTLMS algorithm, we selected $\gamma=0.1 \gamma_{u}, \quad \gamma=\gamma_{u}$ and $\gamma=10 \gamma_{u}$ to simulated The FTLMS algorithm, and compared with the algorithm proposed in this paper. In the low-noise environment, the filter order is converged as shown in Fig. 2, and the additional mean square error EMSE changes as shown in Fig.3.

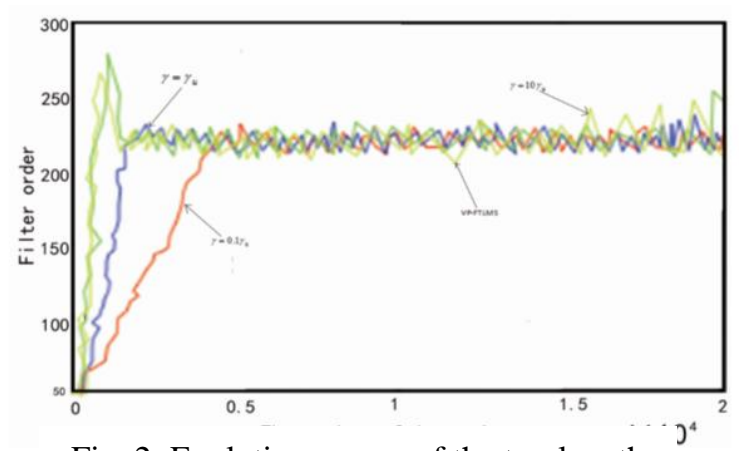

Fig. 2: Evolution curves of the tap-length under low noise condition, $\mathrm{SNR}=20 \mathrm{~dB}$

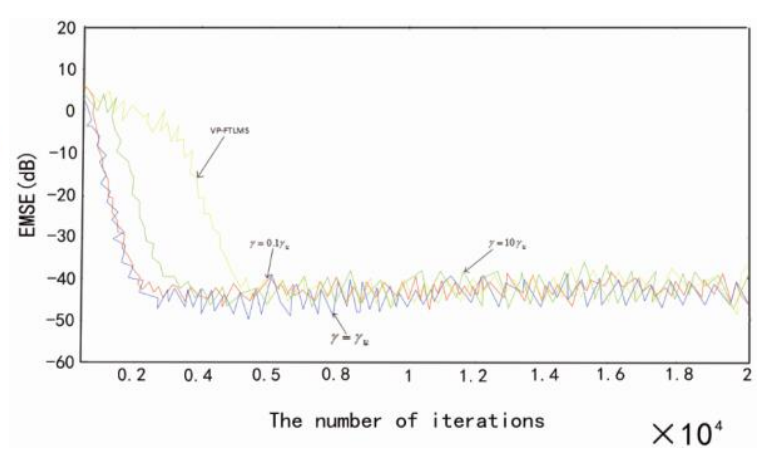

Fig. 3: Evolution curves of the EMSE under low noise condition, $\mathrm{SNR}=20 \mathrm{~dB}$.

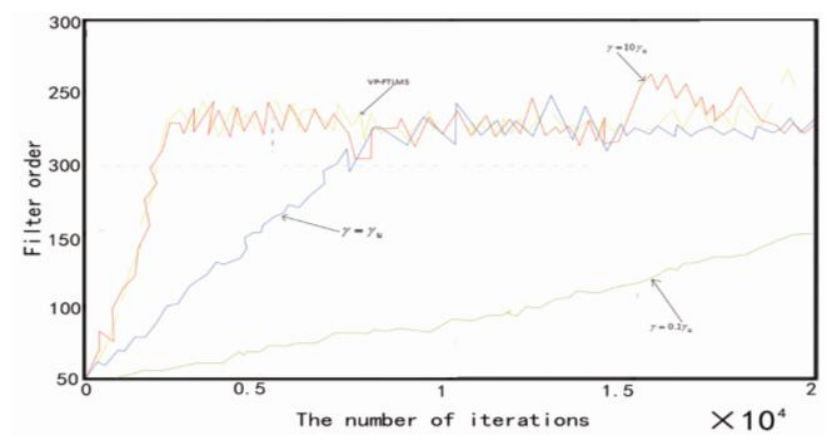

Fig. 4: Evolution curves of the tap-length under high noise condition, SNR=0dB.

\subsection{High Noise, $\mathrm{SNR}=0 \mathrm{~dB}$}

The setting of the high noise environment is similar to that of the low noise environment. The other parameters are the same as those in the low noise environment. The parameter $\delta$ is set to $2, \mu$ ' set to 0.5 , Leakage factor $\alpha$ set to $0.005, \Delta$ set to 20.In the VP-FTLMS algorithm proposed in this paper, smoothing parameter $\rho=0.99$. In order to compare the impact of different values $\gamma$ for the FTLMS algorithm, we selected $\gamma=0.1 \gamma_{u}, \gamma=\gamma_{u}$ and $\gamma=10 \gamma_{u}$ to simulated The FTLMS algorithm. And compared with the algorithm 
proposed in this paper. In the high-noise environment, the filter order is converged as shown in Fig. 4, and the additional mean square error EMSE changes as shown in Fig.5.

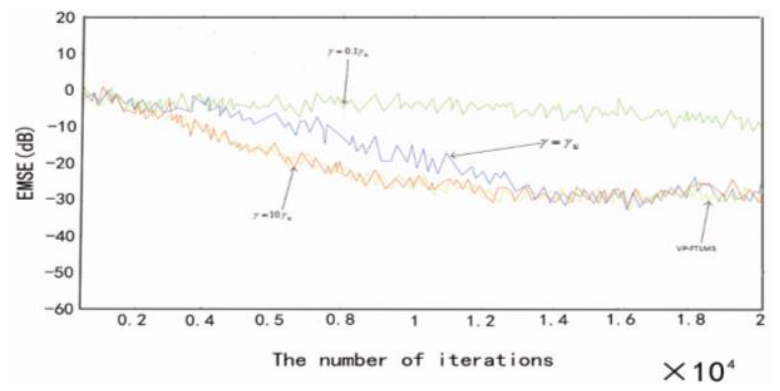

Fig. 5: Evolution curves of the EMSE under high noise condition, $\mathrm{SNR}=0 \mathrm{~dB}$.

\section{Conclusion}

In this paper, a new LMS algorithm with variable degree of iteration parameters is proposed by analyzing the convergence performance of FTLMS algorithm [11]. The simulation results shows that the algorithm proposed in this paper improves the convergence performance of FTLMS algorithm in both low noise and high noise environment, and has faster convergence rate of order, smaller steady state oscillation and smaller extra mean square error, Which solves the contradiction between the convergence speed and the steady state oscillation in the order LMS.

\section{Acknowledgements}

This work is supported by Harbin science and Technology Bureau of youth talent class A innovation in 2015(2015RQQXJ006).

\section{References}

[1] Manpei Tamamura, Eiji Shibata. Application of Active Noise Control for Engine Related Cabin Noise[ J] .JSAE Review, 2006(17): 37-43

[2] Nelson P A. The active minimization of harmonics enclosed sound fields[J].IEEE JSV,2007,117(2):158-163

[3] Zhang Yonggang, Li Ning, Chamber J A, etal. Steady-state performance analysis of a variable tap-length LMS algorithm[J].IEEE Tran Signal Processing,2008,56(2):839-845

[4] Gu Y, Tang K, Cui H. LMS algorithm with gradient descent filter length[J]. IEEE Signal Process Left,2004,11(3):305-307

[5] Dipti Srinivasan, Xin Jin and Ruey Long Cheu. Evalution of Adaptive Neural Network Models for Freeway Incident Detection. IEEE Trans. On Intelligent Transportation System. 2004,5(1):1-11

[6] Hongbin Du, Huihe Shao and Pingjing Yao. Adaptive Neural Network Control for a Class of Low-TriangularStructured Nonlinear Systems. IEEE Transactions on Neural Networks.2006.17(2):509-514

[7] T. Aboulnasr and K. Mayysa. A robust variable step-size LMS-type algorithm: analysis and simulations. IEEE Trans. Signal Processing. 2007,45(3):631-639

[8] Nurgun E, Filiz S. Wavelet transform based adaptive filter analysis and new results. IEEE Trans on SP. 1996,44(9):2163-2168

[9] Zhao Zhicheng, Liu Zhiyuan, Zhang Jinggang. IMC-PID tuning method based on sensitivity specification for process with time delay. Journal of Central South University of Technology,2011,18(4):1153-1160

[10] Sun Yong-wei, Duan Xian-xia, Zhao Wei, et al. Novel variable step-size adaptive LMS time delay estimation algorithm with nonlinear preprocess $[\mathrm{C}] / /$ Proc of the 4th International Congress on Image and Signal Processing. 2011: 2767-2770.

[11] Gong Y, Cowan C F N. A novel variable tap-length algorithm for linear adaptive filter [C] //Proc of IEEE International Conference on Acoustics, Speech, and Signal Proceedings. 2004: 825-828. 